\title{
Elaboration of consensus clinical endpoints to evaluate antimicrobial treatment efficacy in future HABP/VABP clinical trials
}

Emmanuel Weiss ${ }^{1}$, Jean-Ralph Zahar ${ }^{2}$, Jeff Alder $^{3}$, Karim Asehnoune ${ }^{4}$, Matteo Bassetti ${ }^{5}$, Marc J M Bonten $^{6}$, Jean Chastre ${ }^{7}$, Jan De Waele ${ }^{8}$, George Dimopoulos ${ }^{9}$, Philippe Eggimann ${ }^{10}$, Marc Engelhardt $^{11}$, Santiago Ewig ${ }^{12}$, Marin Kollef ${ }^{13}$, Jeffrey Lipman ${ }^{14}$, Carlos Luna ${ }^{15}$, Ignacio MartinLoeches $^{16}$, Leonardo Pagani ${ }^{17}$, Lucy B Palmer ${ }^{18}$, Laurent Papazian ${ }^{19}$, Garyphallia Poulakou ${ }^{20}$, Philippe Prokocimer $^{21}$, Jordi Rello ${ }^{22}$, John H. Rex ${ }^{23}$, Andrew F Shorr ${ }^{24}$, George H Talbot ${ }^{25}$, Visanu Thamlikitkul $^{26}$, Antoni Torres ${ }^{27}$, Richard G Wunderink ${ }^{28}$, and Jean-François Timsit ${ }^{29}$.

${ }^{1}$ Department of anesthesiology and critical care, APHP, Beaujon hospital, Clichy, France; UMR 1149 Centre for research on Inflammation,; Inserm/Université Paris Diderot, F75018, Paris, France;

${ }^{2}$ Jean Ralph Zahar, Department of clinical microbiology and infection control unit, Avicennes hospital, AP-HP, Bobigny, France; IAME, UMR 1137, Université Paris 13, Sorbonne Paris Cité, France.

${ }^{3}$ Jeff Alder, Bayer US LLC, Parsippany, NJ, USA

${ }^{4}$ Karim Asehnoune, University Hospital of Nantes, Intensive Care Unit, Anesthesia and Critical Care Department, Hôtel Dieu, Nantes, France.

(C) The Author(s) 2019. Published by Oxford University Press for the Infectious Diseases Society of America. All rights reserved. For permissions, e-mail: journals.permissions@oup.com. 
${ }^{5}$ Matteo Bassetti, Infectious Diseases Division, Department of Medicine, University of Udine and Santa Maria Misericordia University Hospital, Udine, Italy

${ }^{6}$ Marc J M Bonten, Department of Medical Microbiology \& Julius Center for Health Science and Primary Care, University Medical Center Utrecht, Utrecht, the Netherlands

${ }^{7}$ Jean Chastre, Service de Réanimation Médicale, Institut de Cardiologie, Groupe Hospitalier PitiéSalpêtrière, Assistance Publique-Hôpitaux de Paris, Paris, France.

${ }^{8}$ Jan J. De Waele, Department of Critical Care Medicine, Ghent University Hospital, Ghent, Belgium.

${ }^{9}$ George Dimopoulos MD, PhD, FCCM, FCCP. Department of Critical Care, University Hospital ATTIKON, National and Kapodistrian University of Athens, Greece

${ }^{10}$ Philippe Eggimann, Department of Critical Care, Centre Hospitalier Universitaire Vaudois, Lausanne Switzerland

${ }^{11}$ Marc Engelhardt, Basilea Pharmaceutica International Ltd., Basel, Switzerland

${ }^{12}$ Santiago Ewig, Department of Respiratory Medicine and Infectious Diseases, Evangelic Hospital in Herne and Augusta Hospital in Bochum, Bochum, Germany 
${ }^{13}$ Marin H Kollef, Division of Pulmonary and Critical Care Medicine, Washington University School of Medicine, St. Louis, MO

${ }^{14}$ Jeffrey Lipman, Royal Brisbane and Womens Hospital and The University of Witwatersrand, Brisbane, Australia

${ }^{15}$ Carlos M. Luna, Department of Medicine, Pulmonary Diseases Division, Hospital de Clínicas, Universidad de Buenos Aires, Buenos Aires, Argentina.

${ }^{16}$ Ignacio Martin-Loeches, Department of Clinical Medicine, Multidisciplinary Intensive Care Research Organization (MICRO), St James's Hospital, Trinity Centre for Health Sciences, Dublin, Ireland.

${ }^{17}$ Leonardo Pagani, Infectious Diseases Unit, Bolzano Central Hospital, Bolzano, Italy

${ }^{18}$ Lucy B Palmer, Pulmonary, Critical Care and Sleep Division, SUNY at Stony Brook, HSC T17-040, Stony Brook, NY, USA.

${ }^{19}$ Laurent Papazian, Médecine Intensive-Réanimation, APHM, Hôpital Nord, Chemin des Bourrely, 13015, Marseille, France.

${ }^{20}$ Garyfallia Poulakou, 3rd Department of Medicine, Sotiria General Hospital, Athens, Greece; 
Medical School, National and Kapodistrian University of Athens, Greece

${ }^{21}$ Philippe Prokocimer, Merck \& Co., Inc., Kenilworth, NJ , USA

${ }^{22}$ Jordi Rello, Ciberes, Vall d hebron barcelona hospital campus, Barcelona, Spain

${ }^{23}$ John H. Rex, MD, F2G, Ltd., Eccles, UK

${ }^{24}$ Andrew F. Shorr, Medstar Washington Hospital Center, Washington DC USA

${ }^{25}$ George H Talbot, Talbot Advisors LLC, Anna Maria, FL, USA

${ }^{26}$ Visanu Thamlikitkul, Faculty of Medicine Siriraj Hospital, Mahidol University, Bangkok, Thailand

${ }^{27}$ Antoni Torres. Servei de Pneumologia. Hospital Clinic. Universitat de Barcelona. IDIBAPS.

CIBERES. Barcelona. Spain

${ }^{28}$ Richard G. Wunderink, Northwestern University Feinberg School of Medicine, Chicago IL USA

${ }^{29}$ Jean-François Timsit, 
APHP Medical and Infectious Diseases ICU, Bichat Hospital, Paris, France

UMR 1137 IAME Inserm/Université Paris Diderot, F75018, Paris, France;

\section{*Corresponding author:}

Emmanuel Weiss

Department of anesthesiology and critical care, Beaujon University Hospital, 92110 Clichy

UMR 1149, CRI, Paris Diderot University/ Inserm

Phone: +33140875881

Fax: +33140875610

@: emmanuel.weiss@aphp.fr

\section{Running Title}

Consensus endpoint for HAP/VAP treatment

\section{Summary}

Using the Delphi method, this study provides a multinational expert consensus on separate hierarchical composite endpoints for VABP and HABP, and on a definition of clinical cure that could be considered for use in future HABP/VABP clinical trials. 


\section{Abstract}

Background: Randomized Clinical Trials (RCTs) in Hospital-Acquired (HABP) and VentilatorAssociated Bacterial Pneumonia (VABP) are important for the evaluation of new antimicrobials. However, the heterogeneity in endpoints used in RCTs evaluating treatment of HABP/VABP may puzzle clinicians. The aim of this work was to reach a consensus on clinical endpoints to consider in future clinical trials evaluating antimicrobial treatment efficacy for HABP/VABP.

Methods: Twenty-six international experts from intensive care, infectious diseases and the pharmaceutical industry were polled using the Delphi method.

Results: The panel recommended a hierarchical composite endpoint including, by priority order, for VABP (i) survival at day 28, (ii) mechanical ventilation (MV)-free-days through day 28 , and (iii) clinical cure between study days 7 and 10; and for HABP (i) survival (day 28), and (ii) clinical cure (days 7-10). Clinical cure was defined as the combination of resolution of signs and symptoms present at enrolment and improvement or lack of progression of radiological signs. More than $70 \%$ of the experts agreed to assess survival and MV-free days though day 28, and clinical cure between day 7 and day 10 after treatment initiation. Finally, the hierarchical order of endpoint components was reached after 3 Delphi rounds ( $72 \%$ agreement).

Conclusion: We provide a multinational expert consensus on separate hierarchical composite endpoints for VABP and HABP, and on a definition of clinical cure that could be considered for use in future $\mathrm{HABP} / \mathrm{VABP}$ clinical trials.

\section{Keywords}

Hospital-acquired bacterial pneumonia and ventilator-acquired bacterial pneumonia; Multinational consensus; Delphi method; Hierarchical composite endpoint; Clinical cure 


\section{Introduction}

Hospital-acquired and Ventilator-Associated Bacterial Pneumonia (HABP/VABP) are the second most common nosocomial infections in adults and leading causes of death among critical care infections [1, 2]. Consequently, indications for treatment of HABP and VABP are of considerable interest in the development of new drugs. Nine RCTs of antibiotic therapy for HABP and VABP are currently registered as recruiting patients (clinicaltrials.gov, accessed on January 3rd, 2019). However, a universal, well-accepted and sensitive-to-change criterion for objective and reproducible assessment of drug benefit in HABP/VABP other than all-cause mortality (ACM) at a fixed time (usually 28 days) is still lacking [3, 4]. Appropriate endpoints for HABP/VABP trials have been a subject of debate for several years and remain controversial [3-6].

In the most recent Food and Drug Administration (FDA) guidance for industry on studies evaluating antibiotics for $\mathrm{HABP} / \mathrm{VABP}$ treatment, $\mathrm{ACM}$ at fixed timepoint between 14 and 28 days after therapy start is the accepted primary endpoint [4]. On the other hand, the most recent European Medicines Agency (EMA) guidelines (EMA/CHMP/351889/2013) recommend as the primary endpoint clinical outcome timed from therapy start, so that it occurs 7-14 days after the last possible day of treatment [5]. As shown recently, such a controversy has led to a significant heterogeneity among endpoints reported in RCTs, thereby confounding their results [7]. Based on this uncertainty, our aim was to achieve a consensus on the choice and definition of relevant and plausible primary non-mortality clinical endpoints to address antimicrobial efficacy in HABP/VABP that could be used as a component of future trials. Our approach used the Delphi method [8].

\section{Methods}


A panel of twenty-six international experts from intensive care, infectious diseases and the pharmaceutical industry were consulted using the Delphi method [8] from January 2016 to January 2017 (five questionnaires). More than $70 \%$ of similar answers to a question were necessary to reach a consensus [8]. The iterative process followed is shown in figure 1. More details about initial literature review, expert selection and Delphi process are provided in the supplementary appendix 


\section{Results}

Which endpoint?

We first asked the panelists to rank ten potential primary endpoints commonly reported in the literature [7] by assigning a score from one to ten according to their preference (ten as the best and one as the worst, Figure 1, Table 1). According to $60 \%$ of the experts, clinical cure was the most desirable primary outcome; its mean $( \pm \mathrm{SD})$ ranking was $8.5 \pm 2.7$. Two other endpoints were highly rated: $\mathrm{ACM}$ and mechanical ventilation (MV)-free days, with mean rankings of $7 \pm 2.8$ and $6.5 \pm 2.4$, respectively (Table 1). Ranking of primary endpoints varied according to the specialty and the nationality of the expert: ACM was more frequently chosen as most desirable among non-European experts (55\% vs. $7 \%)$ and/or experts from industry ( $75 \%$ vs $18 \%)$.

Recognizing that several endpoints might be relevant simultaneously, six experts suggested using a composite endpoint and even a hierarchical composite endpoint to combine these items during round one. This idea was approved by $88 \%$ of the panelists who confirmed the three previously mentioned items (i.e. clinical cure, ACM and MV-free days) as the best components of this hierarchical composite endpoint.

Which definition for clinical cure?

Once again, a ranking of the various clinical cure criteria found in the literature was first performed by the experts (Table S1) [7]. Only three items obtained a mean score higher than 3 (across the range 1 to 4) according to the experts: "resolution of signs and symptoms of infection", "improvement of oxygenation parameters" and "no appearance of new signs of sepsis" (Table S1). Given these results, $96 \%$ of the experts agreed to combine "resolution of signs and symptoms of infection", "improvement of oxygenation parameters" and "no appearance of new signs of sepsis" together under the heading 
"Resolution at the end of therapy of signs and symptoms present at enrolment" and to include this item in clinical cure definition ( $88 \%$ agreement). Strikingly, a low score was initially attributed to "the improvement of chest radiography (Table S1)" because the experts thought that a lack of progression of radiographic images could be sufficient to qualify for cure Finally, $72 \%$ of the expert agreed that "resolution or lack of progression of radiological signs of pneumonia" could be part of a clinical cure definition. Altogether, a consensus was reached on a clinical and radiological definition of cure:

1. Clinical: resolution of signs and symptoms present at enrolment

2. Radiography: resolution or lack of progression of radiological signs of pneumonia

To decrease the risk of incorrect pneumonia diagnosis that would impact clinical cure, we next aimed to specify which signs and symptoms should trigger the suspicion of VABP and HABP (Figure 1, Table S2). Interestingly, signs leading to VABP suspicion differed from those leading to HABP suspicion (Table S2). For VABP, four signs were selected by the experts: worsening of gas exchange, hypotension and/or vasopressor requirements, fever or hypothermia, and purulent tracheal secretions (Table S2). For HABP, the panelist added dyspnea and leukocytosis or leukopenia to the VABP suspicion criteria (Table S2). When we asked the experts to confirm these choices, 88 and $73 \%$ of them agreed for VABP and HABP suspicion criteria respectively.

Which time-point for endpoint assessment?

In parallel, the optimal timeframe for endpoint assessment was selected: expert choices were day 28 for ACM, the period from the diagnosis to day 28 for MV-free days, and the period between days 7 and 10 after treatment initiation for clinical cure. More details on expert choices regarding timepoints are provided in supplementary results. 
Consensus hierarchy between components of a possible composite endpoint.

When considered together, the 3 endpoints of ACM at day 28, MV-free days from diagnosis to day 28, and clinical cure between study days 7 and 10 seemed sufficiently independent as to suggest a possible composite endpoint. The panel was thus asked to rank these three items according to their importance. While ACM was the most important component for $72 \%$ of the experts, the relative ranking of the two remaining components was more difficult to establish: $60 \%$ of the experts placed clinical cure in the second position, whereas $40 \%$ of them thought that MV-free days was more important for this rank. To address this question, we used two small case-vignettes reflecting the possible situations and asked the experts which is most desirable: 1) a survivor at day 28 , not cured 7 to 10 days after treatment initiation, and who required a short duration of MV before day 28 or 2) a survivor at day 28 clinically, cured 7 to 10 days after treatment initiation and who required a long duration of MV before day 28 . Using this strategy, $75 \%$ of the experts judged that the first situation with short duration of MV was the more desirable scenario, demonstrating that the panel valued MV-free days as more important than time to clinical cure.

Of note, some experts suggested differentiating HABP from VABP and $85 \%$ of the panel agreed to a dual composite endpoint for non-ventilated HABP consisted of ACM at day 28 and clinical cure 7 to 10 days after treatment initiation (Figure 2). Conversely, in cases of HABP requiring MV (i.e., ventilated HABP) experts recommended adopting the same rules as for VABP.

Finally, some experts suggested a discrepancy in the direction of the composite endpoint that included $\mathrm{ACM}$ as a negative endpoint and MV-free days and clinical cure as positive ones. To cope with that situation, $96 \%$ of the experts agreed to revise this endpoint to "survival at day 28 ".

In conclusion, we reached a consensus providing separate hierarchical composite endpoints for VABP and HABP that assess by priority order: 1 . Day-28 survival, 2. Any number of MV-free days from the 
diagnosis of pneumonia to day 28 (only for VABP), and 3. Clinical cure measured between study days 7 and 10 (Figure 2). As an example, we ranked the outcome of four representative patients according to five different primary endpoints including our hierarchical composite endpoint (Figure 3) and found good discriminatory ability of the latter. 


\section{Discussion}

Several studies are currently investigating the efficacy of new antimicrobials against multidrugresistant pathogens or will do so in the near future. Given their frequency, HABP and VABP are key testing grounds for new agents against Gram-negative pathogens, despite the lack of validated nonmortality, patient- and physician-centered clinical endpoints, which would specifically assess the effectiveness of a new drug. Using the Delphi method to elicit opinions from a panel of 26 international experts, we have developed consensus clinical endpoints with the potential of the evaluation of antimicrobial treatment for HABP/VABP. This carefully implemented iterative process required a notable effort of the panel to obtain a hierarchical composite endpoint that includes survival, MV-free days and clinical cure and should provide a better discrimination of patient outcomes.

Taken separately, each component of this composite endpoint has strengths and weaknesses. A high agreement was obtained regarding the inclusion of ACM (or survival) as an outcome measure. As suggested by the FDA, ACM offers numerous advantages: it is verifiable, reproducible, and it is the only unambiguously and entirely objective "patient-centered" outcome [3, 4, 9]. However, the EMA raised arguments against it, for the reason that a substantial part of ACM (or survival) is not related to HABP/VABP in ICU but rather to underlying disease. $[3,5]$.

The second criterion selected by the experts was ventilator-free days. This patient-oriented endpoint is easy to measure but remains susceptible to unrelated events. While its impact on patient's benefit is unambiguous, the process around discontinuing MV includes a subjective judgment by the care provider. 
Clinical cure, the last criterion included in our composite, is disease-oriented [9]. While very useful (especially if mortality is low), it is prone to subjective clinical considerations and geographic or practice-setting variability. Acknowledging the lack of a validated clinical cure definition as an important drawback, we developed a consensus definition of this endpoint and then validated a list of signs and symptoms of HABP and/or VABP that should be resolved to qualify for cure.

Besides ACM, MV-free days and clinical cure, several other outcomes may have deserved to be included in the composite endpoint but were not suggested or chosen by the panelists. Among them, complications of the disease are of particular interest because they reflect how patients feel and function and because better tolerability may confer an advantage to a new treatment [10]. CPIS acceptance was very variable, with $19 \%$ of the panel considering it a highly desirable outcome measure, while $30 \%$ judging it as not useful at all. [1,2]. The experts also very readily rejected microbiological cure, probably because of the lack of correlation with patient outcome. Finally, some medium- to long-term patient-centered outcomes, such as quality of life or hospital discharge [11], were not suggested as they were deemed difficult to measure.

The consensus composite endpoint that we provide includes the three most significant outcomes according to the experts. As shown with Figure 3 case vignettes, reporting of a composite may improve the assessment of patient's outcome compared to single endpoint. However, an inherent limitation of composite endpoints is that it emphasizes each patient's first event, which is often the outcome of lesser importance. For example, a patient who was cured at day 7 but died during his ICU stay could be considered as having a better outcome than a second patient who was discharged alive from the ICU but was cured from his VABP later (Figure 3). Here, as previously suggested by Pocock et al. [12] with the win ratio, and Evans et al. [13] with the Desirability Of Outcome Ranking concept, we introduced a hierarchy to take the different priorities of the components into account and allow a better patient differentiation according to the desirability of the outcome. Those hierarchical composite endpoints were recently used in several studies comparing different antibiotic strategies in 
community-acquired pneumonia [14], intra-abdominal infections [15] or mixed infections caused by carbapenem-resistant Enterobacteriaceae [11]. However, statistical design/analysis have to be anticipated [16] to operationalize the use of the rank-based composite endpoint [17]. While conventional composite could increase power by combining some or all variables included in the hierarchy as a primary endpoint, a rank-based composite (as suggested here) would test the components of the primary endpoint in a prespecified, hierarchical order with termination rules (depending on null hypothesis rejection) of the procedure at each step. In this case, multistate models or hierarchical nested-trial designs should be considered [16].

Several areas of uncertainty remain. First, the debate about the relative merits of utilizing clinical cure or ACM remains unresolved. The RCTs assessing antibiotic therapy for HABP and VABP currently reported as recruiting on www.clinicaltrials.gov illustrate this uncertainty with ACM and clinical cure respectively chosen as primary endpoint in two third and one third of cases. In our study, although the sample size may limit the meaning of the results, clinical cure seemed to be especially recommended by European experts consistent with EMA recommendations [5], while others, including experts from the pharmaceutical industry, showed a preference for ACM, consistent with FDA guidance [4].

The timepoint to assess each component of the proposed composite endpoint was difficult to define. While a consensus was rapidly reached for ACM, probably because choosing a late timepoint (day 28) increases event number [10], it required two rounds for MV-free days. The highest difficulties were encountered in clinical cure, for which the experts were divided between early and late timepoints, requiring 3 Delphi rounds to reach a consensus about day 7 to day 10 after treatment initiation, with only $74 \%$ of similar answers. Such uncertainty reflects the opaqueness of our understanding of "clinical cure". Of note, an early timepoint reflecting initial response to treatment was added beside the later timepoints used for $\mathrm{ACM}$ and $\mathrm{MV}$-free days that may be more representative of global outcome. In addition, this timepoint was earlier than the one chosen for clinical cure evaluation by regulatory agencies ( 7 to 14 days after antibacterial treatment completion). 
Whether treatment efficacy of HABP, ventilated-HABP and VABP could be studied together remains debated [10]. Although combining these entities is convenient for analyzing data (e.g. by ensuring an adequate power), differences in outcome between these subcategories may preclude such pooling. In this work, while the experts considered $\mathrm{HABP}$ requiring MV as equivalent to $\mathrm{VABP}$, the majority of them suggested a specific endpoint for non-ventilated HABP. This separation is consistent with a recent statement from the FNIH [10]. Based on the analysis of ten contemporary HABP/VABP clinical trials, the authors found that the ACM rates are similar for ventilated HABP and VABP, and higher than for non-ventilated HABP, suggesting inclusion of ventilated HABP and VABP in the same clinical trial. Similarly, suspicion criteria for non-ventilated HABP differed from those of the two other entities. Despite variability in expert opinion, a large majority of them agreed with four VABP suspicion criteria (gas exchange worsening, purulent tracheal secretions, hypotension and/or vasopressor requirements, and fever or hypothermia) and six HABP suspicion criteria. Notably, hypotension and/or vasopressor requirements were added to VABP suspicion criteria, whereas they are not included in "usual" criteria recommended by the Infectious Disease Society of America and American Thoracic Society [1]. These findings suggest that in case of severe ICU-acquired sepsis, VABP should be systematically suspected given its frequency. Conversely, leukocyte count was removed from VABP suspicion criteria, probably because of the numerous factors that could affect this inflammatory parameter in ICU.

Some limitations of our novel endpoint have to be underlined. Consensus on the hierarchical ranking of the elements of the composite was difficult to obtain. While the experts gave the maximum weight to ACM, three Delphi rounds and the use of case vignettes were required to choose the more clinically meaningful outcome between MV-free days and clinical cure, with a final panel agreement limited to $75 \%$. Such difficulties in ranking of the components demonstrate potential subjectivity in expert opinion, given the paucity of evidence, which was not explained by expert background. Furthermore, consensus-obtained timepoints for endpoint assessment were difficult to obtain, especially for clinical cure, which was a source of intense debate between clinicians and experts from the pharmaceutical industry. Another possibility would have been to study the kinetics of disease progression instead of a 
binary cure/non-cure at a fixed timepoint. In particular, multistate endpoints could be used to demonstrate a treatment effect over the complete treatment process instead of merely at the end of follow-up [16]. The choice of the statistical design (i.e. superiority or non-inferiority), which was not predetermined, may also change expert opinion about endpoints. Use of the composite endpoint in non-inferiority trials will require assessment of its effect size relative to effective therapy. Concerning our panel that included both industry and academic experts in the field, a third group composed of patient representatives would have been interesting. Including regulatory agencies representative was also considered but was not retained to avoid potential already formed ideas or conflicts of interest. Finally, as for any new method, the use of such endpoint may be initially limited by lack of familiarity among trialists.

In conclusion, judgment-based criteria for assessing antimicrobial efficacy in HABP/VABP are heterogenous. Therefore, a convergence on approaches for the evaluation of antibacterial drugs is urgently required [18]. We reached a consensus on a set of hierarchical composite endpoints for VABP and HABP using the Delphi method by drawing on the expertise of 26 international experts. A pilot validation of our proposed endpoint by use of data from an already completed clinical trial, which was conducted with the classic endpoints, should be performed first. Then, if validated, it could be considered for inclusion in future trials and, if further validated, may allow a pragmatic and homogenous assessment of antimicrobial efficacy in future HABP/VABP RCTs. 


\section{Acknowledgments}

The authors thank Celine Feger, MD (EMIBiotech) for her editorial support.

\section{Funding}

This research was supported by the Innovative Medicines Initiative Joint Undertaking under grant agreement n ${ }^{\circ} 115523$ [Combatting Bacterial Resistance in Europe - COMBACTE], resources of which are composed of financial contribution from the European Union's 7th Framework Programme (FP7/2007-2013) and EFPIA companies' in kind contribution.

\section{Conflict of interest statements}

Dr. Talbot reports payments from Adynxx, Anacor Pharmaceuticals, Meiji-Seika, Nabriva Therapeutics, Polyphor Ltd, Zavante Therapeutics, AN2 Therapeutics, Recida, Actelion, and Tripex, all outside the submitted work. Dr. Asehnoune reports personal fees from Baxter, Fresenius, LFB, Fisher and Payckel, and grants from Aridis, outside the submitted work. Dr. Weiss reports personal fees from MSD, Baxter, Gilead, and Biomerieux, outside the submitted work. Dr. Timsit reports grants and personal fees from Pfizer and Merck, personal fees from 3M, personal fees from Biomerieux, personal fees from Gilead, and personal fees from Maat Pharma, outside the submitted work. Dr. Zahar reports payments from Pfizer, 3M, Merck, and Eumedica, outside the submitted work. Dr. De Waele reports grants from Research Foundation Flanders, during the conduct of the study; and consultancy fees from AtoxBio, and MSD, Pfizer, Bayer, and Accelerate, outside the submitted work. Dr. Bassetti reports grants and personal fees from Pfizer and MSD, and personal fees from Menarini, Angelini, Bayer, and Thermofisher, outside the submitted work. Dr. Chastre reports personal fees from Bayer, Pfizer, AstraZeneca/Medimmune, Inotrem, Kenta/Aridis, Tigenix, and Accelerate Diagnostics, outside the submitted work. Dr. Poulakou reports grants and personal fees from Pfizer and personal fees from MSD, outside the submitted work. Dr. Lipman reports personal fees from Bayer ESICM Advisory Board, MSD Antibacterials Advisory Board, Pfizer South Africa, MSD South Africa, and Pfizer International, outside the submitted work. Dr. Rello reports personal fees from Pfizer, Anchoagen, and Roche, and grants from Bayer, outside the submitted work. Dr. Rex reports 
consulting fees from Phico Therapeutics; ABAC Therapeutics; Polyphor, Ltd.; Heptares Therapeutics, Ltd.; Gangagen, Ltd.; Meiji Seika Pharma; Basilea Pharmaceutica International Ltd.; Allecra Therapeutics GmbH; Forge Therapeutics, Inc.; SinSa Labs; AtoxBio; Peptilogics; F. HoffmannLaRoche, Ltd.; and Novo Holdings; he is a ahareholder in AstraZeneca Pharmaceuticals; F2G, Ltd; Adenium Biotech ApS; Advent Life Sciences; Macrolide Pharmaceuticals; and Bugworks Research, Inc. All other authors declare they have no conflict of interest relevant to the present work. 


\section{References}

1. Kalil AC, Metersky ML, Klompas M, et al. Management of Adults With Hospital-acquired and Ventilator-associated Pneumonia: 2016 Clinical Practice Guidelines by the Infectious Diseases Society of America and the American Thoracic Society. Clin Infect Dis 2016; 63(5): e61-e111.

2. Torres A, Niederman MS, Chastre J, et al. International ERS/ESICM/ESCMID/ALAT guidelines for the management of hospital-acquired pneumonia and ventilator-associated pneumonia: Guidelines for the management of hospital-acquired pneumonia (HAP)/ventilatorassociated pneumonia (VAP) of the European Respiratory Society (ERS), European Society of Intensive Care Medicine (ESICM), European Society of Clinical Microbiology and Infectious Diseases (ESCMID) and Asociacion Latinoamericana del Torax (ALAT). Eur Respir J 2017; 50(3).

3. Timsit JF, de Kraker MEA, Sommer H, et al. Appropriate endpoints for evaluation of new antibiotic therapies for severe infections: a perspective from COMBACTE's STAT-Net. Intensive Care Med 2017; 43(7): 1002-12.

4. Guidance for Industry Hospital-Acquired Bacterial Pneumonia and Ventilator- Associated Bacterial Pneumonia: Developing Drugs for Treatment: U.S. Department of Health and Human Services Food and Drug Administration Center for Drug Evaluation and Research (CDER), 2014.

5. Addendum to the guideline on the evaluation of medicinal products indicated for treatment of bacterial infections: European Medical Agency, 2013.

6. Rex JH, Talbot GH, Goldberger MJ, et al. Progress in the Fight Against Multidrug-Resistant Bacteria 2005-2016: Modern Noninferiority Trial Designs Enable Antibiotic Development in Advance of Epidemic Bacterial Resistance. Clin Infect Dis 2017; 65(1): 141-6.

7. Weiss E, Essaied W, Adrie C, Zahar JR, Timsit JF. Treatment of severe hospital-acquired and ventilator-associated pneumonia: a systematic review of inclusion and judgment criteria used in randomized controlled trials. Crit Care 2017; 21(1): 162.

8. Okoli C PSD. The Delphi method as a research tool: An example, design considerations and applications. Information and Management 2004; 42: 15-29.

9. de Grooth HJ, Parienti JJ, Oudemans-van Straaten HM. Should we rely on trials with diseaserather than patient-oriented endpoints? Intensive Care Med 2017.

10. group FftNIoHBCHVw. Considerations for clinical trial design for the studt of hospitalacquired bacterial pneumonia and ventilator-associated bacterial pneumonia. For submission to docket \#FDA-2013-N-0556. https://www.regulations.gov/document?D=FDA-2010-D0589-0027, 201726 May 2017.

11. van Duin D, Lok JJ, Earley M, et al. Colistin vs. Ceftazidime-avibactam in the Treatment of Infections due to Carbapenem-Resistant Enterobacteriaceae. Clin Infect Dis 2017. 
12. Pocock SJ, Ariti CA, Collier TJ, Wang D. The win ratio: a new approach to the analysis of composite endpoints in clinical trials based on clinical priorities. Eur Heart J 2012; 33(2): 17682 .

13. Evans SR, Rubin D, Follmann D, et al. Desirability of Outcome Ranking (DOOR) and Response Adjusted for Duration of Antibiotic Risk (RADAR). Clin Infect Dis 2015; 61(5): $800-6$.

14. Schweitzer VA, van Smeden M, Postma DF, Oosterheert JJ, Bonten MJM, van Werkhoven $\mathrm{CH}$. Response Adjusted for Days of Antibiotic Risk (RADAR): evaluation of a novel method to compare strategies to optimize antibiotic use. Clin Microbiol Infect 2017; 23(12): 980-5.

15. Celestin AR, Odom SR, Angelidou K, et al. Novel Method Suggests Global Superiority of Short-Duration Antibiotics for Intra-abdominal Infections. Clin Infect Dis 2017; 65(9): 15779.

16. de Kraker MEA, Sommer H, de Velde F, et al. Optimizing the Design and Analysis of Clinical Trials for Antibacterials Against Multidrug-resistant Organisms: A White Paper From COMBACTE's STAT-Net. Clin Infect Dis 2018.

17. Schaller SJ, Anstey M, Blobner M, et al. Early, goal-directed mobilisation in the surgical intensive care unit: a randomised controlled trial. Lancet 2016; 388(10052): 1377-88.

18. agency Em. Tripartite meeting held between the PMDA, EMA, and FDA in Kyoto, Japan to discuss convergence on approaches for the evaluation of antibacterial drugs. 2017.

19. Luna CM, Blanzaco D, Niederman MS, et al. Resolution of ventilator-associated pneumonia: prospective evaluation of the clinical pulmonary infection score as an early clinical predictor of outcome. Crit Care Med 2003; 31(3): 676-82.

20. Vincent JL, Moreno R, Takala J, et al. The SOFA (Sepsis-related Organ Failure Assessment) score to describe organ dysfunction/failure. On behalf of the Working Group on SepsisRelated Problems of the European Society of Intensive Care Medicine. Intensive Care Med 1996; 22(7): 707-10.

21. Knaus WA, Zimmerman JE, Wagner DP, Draper EA, Lawrence DE. APACHE-acute physiology and chronic health evaluation: a physiologically based classification system. Crit Care Med 1981; 9(8): 591-7.

22. Le Gall JR, Lemeshow S, Saulnier F. A new Simplified Acute Physiology Score (SAPS II) based on a European/North American multicenter study. JAMA 1993; 270(24): 2957-63. 


\section{Figure legends}

Figure 1. Iterative process followed to achieve the proposed hierarchical composite endpoint

Figure 2. Final hierarchical composite endpoint proposed

Figure 3. Ranking of desirability of the outcome of four representative patients according to the chosen primary endpoint.

Patient outcomes were ranked from most desirable (first position of the podium) to least desirable (third position of the podium or even outside the podium). Classical composite endpoint: No hierarchy between components (ACM at day 28, MV-free day from diagnosis to day 28, and clinical cure within 7-10 days of study initiation); The earliest event goes first. Hierarchical composite endpoint: ACM at day 28 is firstly assessed. Then, MV-free day from diagnosis to day 28 is assessed. Finally, clinical cure within 7-10 days of study initiation. 
Table 1. Ranking of endpoints found in the literature by the experts.

\begin{tabular}{|c|c|c|}
\hline $\operatorname{Rank}^{1}$ & Item & $\begin{array}{l}\text { Mean }( \pm \mathrm{SD}) \text { score } \\
\text { (over ten) }\end{array}$ \\
\hline 1 & Clinical Cure & $8.5 \pm 2.7$ \\
\hline 2 & $\mathrm{ACM}$ & $7.0 \pm 2.8$ \\
\hline 3 & MV Free-Days & $6.5 \pm 2.4$ \\
\hline 4 & Improvement in Oxygenation Parameters & $5.8 \pm 2.4$ \\
\hline 5 & Number of Days Before Resolution & $5.6 \pm 2.4$ \\
\hline 6 & CPIS $^{2}$ decrease & $5.2 \pm 2.4$ \\
\hline 7 & Microbiological Cure & $5.1 \pm 2.4$ \\
\hline 8 & Safety & $3.8 \pm 2.4$ \\
\hline 9 & $\mathrm{PCT}^{3}$ Decrease & $3.7 \pm 3.1$ \\
\hline 10 & Acquisition of Antimicrobial Resistance & $3.0 \pm 2.3$ \\
\hline
\end{tabular}


${ }^{1}$ A score from one to ten was attributed to every criterion by each expert according to their preference (ten as the best and one as the worst). Results are given as mean $( \pm \mathrm{SD})$ score obtained by each endpoint.

${ }^{2}$ CPIS: Clinical pulmonary infection score (CPIS) [19]

\section{${ }^{3}$ Procalcitonin}


Figure 1

\begin{tabular}{|c|c|c|c|c|}
\hline \multirow[b]{2}{*}{ 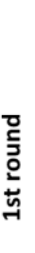 } & $\begin{array}{l}\begin{array}{l}\text { Endpoint } \\
\text { components }\end{array} \\
\underline{\text { nat }}\end{array}$ & $\frac{\text { Assessment }}{\text { timepoint }}$ & Cure definition & $\frac{\text { HABP/VABP }}{\text { suspicion criteria }}$ \\
\hline & $\begin{array}{l}\text { Ranking of most } \\
\text { frequent endpoints } \\
\text { used in RCTs } \\
\text { according to expert } \\
\text { preferences }\end{array}$ & & $\begin{array}{l}\text { Ranking of most } \\
\text { frequent clinical cure } \\
\text { criteria used in RCTs } \\
\text { according to expert } \\
\text { preferences }\end{array}$ & \\
\hline & $\downarrow$ & & $\downarrow$ & \\
\hline 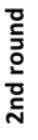 & $\begin{array}{c}\text { Construction of a } \\
\text { composite endpoint } \\
\text { including highly rated } \\
\text { endpoints }\end{array}$ & $\begin{array}{l}\text { Ranking of the most } \\
\text { frequent timepoints }\end{array}$ & $\begin{array}{l}\text { Construction of a } \\
\text { clinical cure } \\
\text { definition including } \\
\text { highly rated criteria }\end{array}$ & \\
\hline 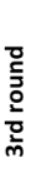 & $\begin{array}{c}\downarrow \\
\text { Assessment of the } \\
\text { proposed composite } \\
\text { endpoint by the } \\
\text { experts }\end{array}$ & $\begin{array}{l}\text { used to assess each } \\
\text { endpoint of interest } \\
\text { in RCTs }\end{array}$ & Assessment of the & $\begin{array}{l}\text { Ranking of HABP/VABP } \\
\text { suspicion criteria } \\
\text { according to expert } \\
\text { preferences }\end{array}$ \\
\hline & $\downarrow$ & $\downarrow$ & proposed clinical cure & $\downarrow$ \\
\hline 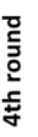 & $\begin{array}{l}\text { Prioritization within } \\
\text { endpoint component } \\
\text { using case vignettes }\end{array}$ & $\begin{array}{l}\text { Assessment of the } \\
\text { best timepoint to } \\
\text { assess each endpoint } \\
\text { of the composite }\end{array}$ & $\begin{array}{l}\text { derinition by the } \\
\text { experts }\end{array}$ & $\begin{array}{l}\text { Assessment of the } \\
\text { proposed signs that } \\
\text { should trigger VAP } \\
\text { and HAP suspcion }\end{array}$ \\
\hline & & & & \\
\hline & & ent of a consens & erarchical composit & ndpoint \\
\hline
\end{tabular}


Figure 2 
VABP

and ventilated $H A B P$

1) Survival at day 28

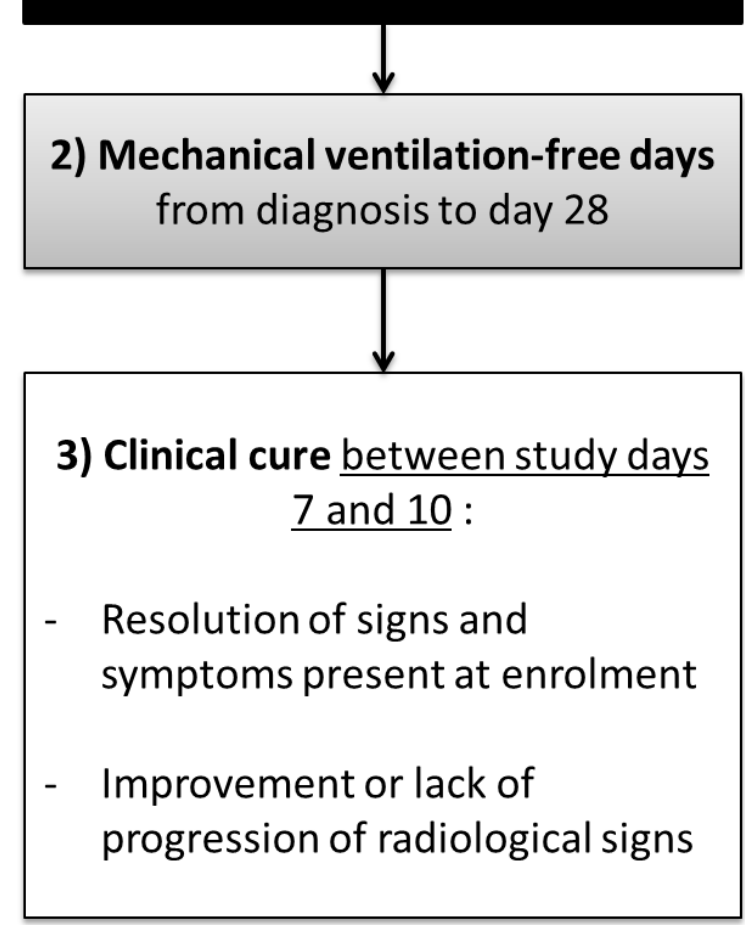

HABP

1) Survival at day 28

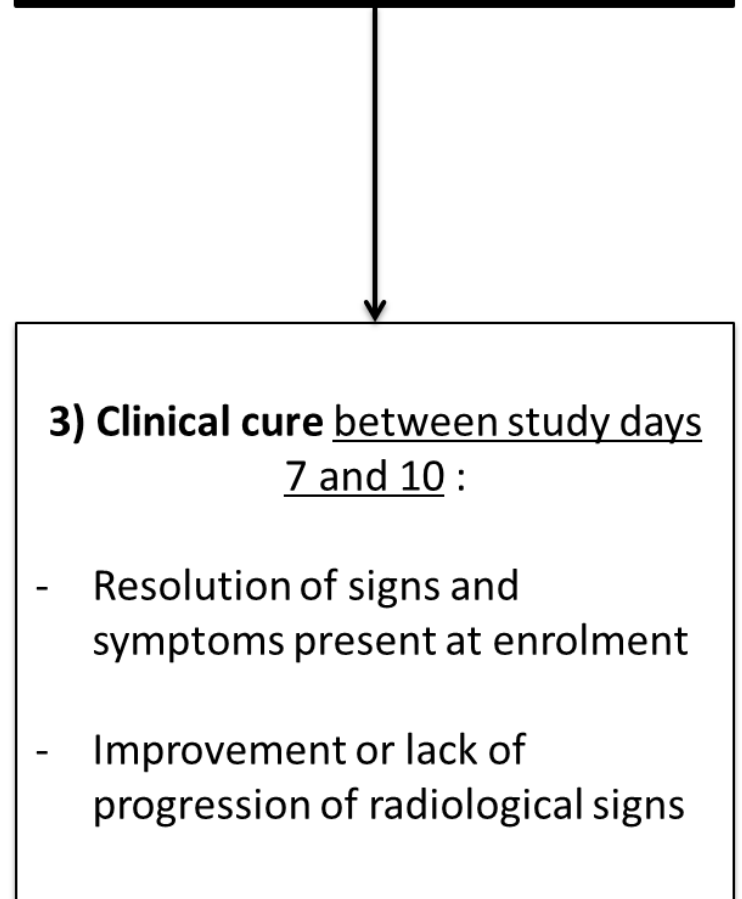


Figure 3

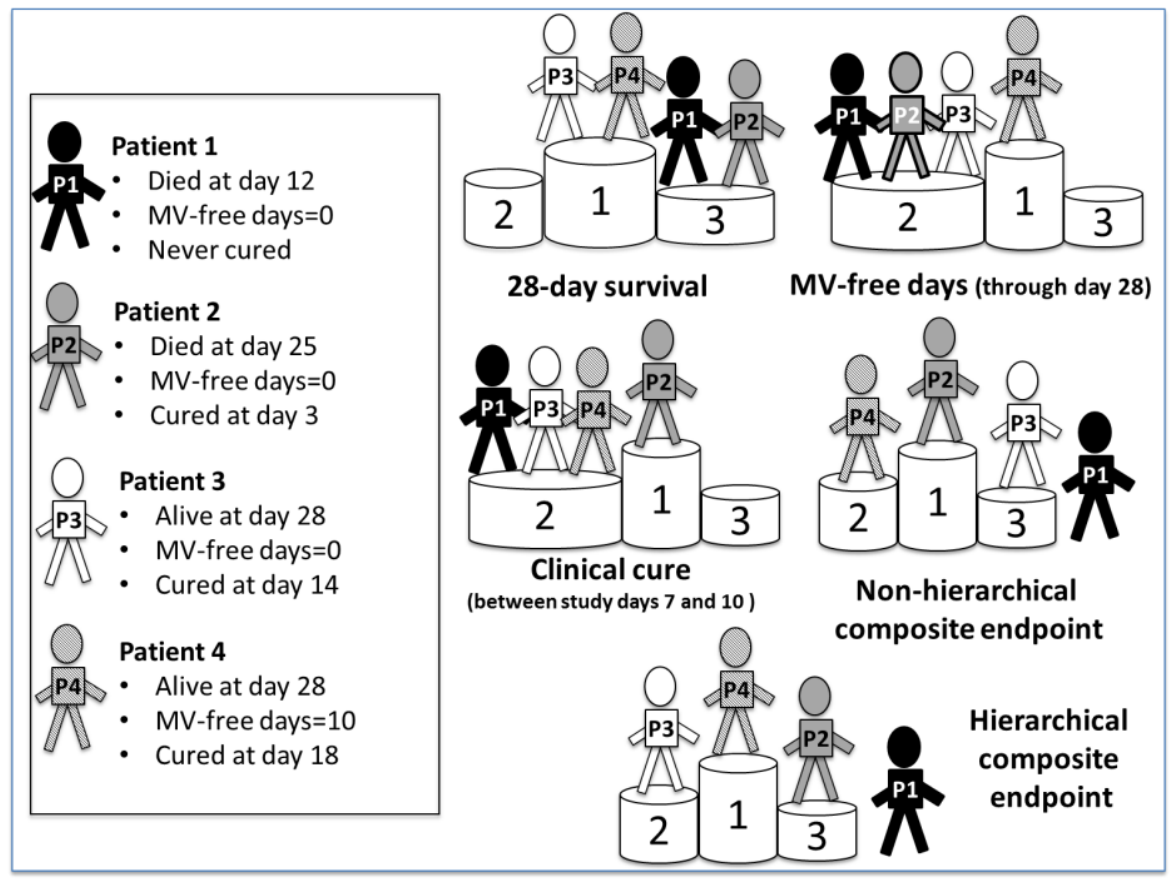

